\title{
Guided-Mode Resonance Filter With ULTRA-NARROW BANDWIDTH OVER THE VISIBLE FREQUENCIES FOR LABEL-FREE OPTICAL BIOSENSOR
}

\author{
Phuc Toan DANG $G^{1,2, *}$, Khai Q. LE $E^{1,2}$, Quang Minh NGO $O^{3,4}$, H. P. T. NGUYEN ${ }^{5}$, \\ Truong Khang NGUYEN ${ }^{1,2, *}$
}

\author{
${ }^{1}$ Division of Computational Physics, Institute for Computational Science, Ton Duc Thang \\ University, Ho Chi Minh City, Vietnam \\ ${ }^{2}$ Faculty of Electrical and Electronics Engineering, Ton Duc Thang University, Ho Chi Minh \\ City, Vietnam \\ ${ }^{3}$ University of Science and Technology of Hanoi, Vietnam Academy of Science and Technology, 18 \\ Hoang Quoc Viet, Cau Giay, Hanoi, Vietnam \\ ${ }^{4}$ Graduate University of Science and Technology, Vietnam Academy of Science and Technology, \\ 18 Hoang Quoc Viet, Cau Giay, Hanoi, Vietnam \\ ${ }^{5}$ Department of Electrical and Computer Engineering, New Jersey Institute of Technology, \\ University Heights, Newark, NJ 07102, USA
}

*Corresponding Authors: Phuc Toan Dang (email: dangphuctoan@tdtu.edu.vn); Truong Khang

Nguyen (email: nguyentruongkhang@tdtu.edu.vn)

(Received: 18-February-2019; accepted: 13-May-2019; published: 30-June-2019)

DOI: http://dx.doi.org/10.25073/jaec.201932.233

\begin{abstract}
A practical guided-mode resonance filter operating in the visible band of the electromagnetic spectrum is numerically designed in this paper. The filter provides high background transmission (>90\%) with almost perfect reflection at resonance wavelengths of $623 \mathrm{~nm}$ and 641 nm for TE and TM modes, respectively. Our filter is also characterized by its sensitivity to incident angles, polarizations, and a refractive index of the surrounding environment which are utilized in practical applications such as tunable optical filters, imaging or detection. We show that the resonant transmission spectral response can be used for highly sensitive, a potential labelfree refractive index biosensor having sensitivities of $90 \mathrm{~nm} / R I U$ and $103 \mathrm{~nm} / \mathrm{RIU}$, and figure of merits of 1.93 and 2.13 for TM and TE polarizations, respectively.
\end{abstract}

\section{Keywords}

Guided-mode Resonance, Filter, Visible, Narrow Band.

\section{INTRODUCTION}

Guided-mode resonance (GMR) or waveguidemode resonance is known as a phenomenon in which the resonant waveguide modes are excited in phase-matching elements such as slab waveguide gratings and photonic crystal slabs [1]. GMR gratings and photonic crystal slabs are usually used for optical filtering application thanks to their unique spectral response. A typical GMR grating filter includes a stack of thin dielectric material layers with gratings/photonic crystals inscribed on the waveguiding layer to 
support guided modes which resonantly results in high reflection and near-zero transmission at the corresponding resonant wavelengths [2-6]. GMR effect arises as an evanescent diffraction phenomenon occurring at an interface between gratings and free-space when an incident light is coupled into the guided mode of the waveguide component and propagates in it at specific optical parameters of wavelength, angle and polarization modes of the incident light $[5,6]$. GMR filters might have many useful characteristics which include narrow band, high peak efficiency, flexible structures [2], [7-11], etc. Therefore, they have been widely studied for filter applications with practical demands such as narrow band, total reflection, and the others [12, 13]. Normally, a high-index contrast grating structure is used for these applications since it has low-lost dielectric thanks to a combined architecture between a high index material grating and low index materials [14]. Besides, photonic crystal structures implemented in planar waveguides is also preferred because of its high quality (Q) factor property [15].

GMR filters present a highly-sensitive property to optical parameters of their structural geometry and conditions of the incident light. Particularly, the angular sensitivity will lead to the spectral location sensitivity in the bandwidth range so that it can be used effectively to adjust the central transmission dips of the filter to the desired wavelength. Therefore, it can be used to design tunable optical filters in both their resonant wavelengths and $Q$-factors [2], [16]. Apart from filtering applications, we have recently employed GMR-based gratings/photonic crystal slabs for optical switching/bistability applications. We introduced innovative all-optical switching devices with low switching power and high bistability efficiency thanks to the induced GMR in the gratings and photonic crystal slab waveguides [17]. In addition, one of the essential characteristics of GMR filters is its high sensitivity to refractive index changes in the surrounding environment of the high-index waveguiding layer. Therefore, guided-mode resonance filters have been increasingly utilized for sensing applications [18], [19].

In this paper, we numerically design and characterize an ultra-narrowband GMR filter oper- ating in the visible band of the electromagnetic spectrum. Angular and polarization of incident light and surrounding environment influence on the GMR filter will comprehensively be analyzed in this study. We observe a stop-band which blocks the impinging light over a narrow bandwidth of frequencies in the visible and passes all remaining frequencies beyond the band-stop, which plays an important role in various imaging or detection applications [20]. In addition, we investigate the refractive index sensing performance of the GMR filter for both transverse electric (TE) and transverse magnetic (TM) modes. The resulting high sensitivity and selectivity of the filter to a refractive index change of the surrounding environment provide possibilities to realize high-efficiency integrated on-chip labelfree optical biosensors. All simulations are performed by using the commercial electromagnetic simulation CST MICROWAVE STUDIO (CST MWS) package [21].

\section{GMR FILTER DESIGN}

Fig. 1 shows a schematic illustration of the proposed GMR filter which consists of a $\mathrm{Ta}_{2} \mathrm{O}_{5}$ waveguiding layer with patterned gratings positioned on a glass substrate via a 10-nm-thick adhesion $\mathrm{SiO}_{2}$ layer. All materials with dispersive properties are extracted from the material library of the simulation software [21]. The wavelength dependent refractive indices of the materials were taken from the literature. We start our investigation with a designed GMR filter having a transmission resonance in the visible. We have studied many parameters and found that the periodicity affected the resonance wavelengths significantly. Other parameters such as $h, W, d_{g}$ were used to optimize the reflection dip and transmission background at the resonance wavelength. The design utilizes a total $\mathrm{Ta}_{2} \mathrm{O}_{5}$ thickness of $h=0.1 \mu \mathrm{m}$, a grating depth, pitch and width of $d_{g}=0.08 \mu \mathrm{m}, P=0.49 \mu \mathrm{m}$, and $W=0.16 \mu \mathrm{m}$, respectively. The wavelength dependent refractive indices of glass, adhesion, and homogeneous $\mathrm{Ta}_{2} \mathrm{O}_{5}$ layers are given as $\mathrm{n}_{s}, \mathrm{n}_{a}$, and $\mathrm{n}_{w}$, respectively.

To achieve resonance, waveguide modes have to be generated with the incident wave satisfy- 


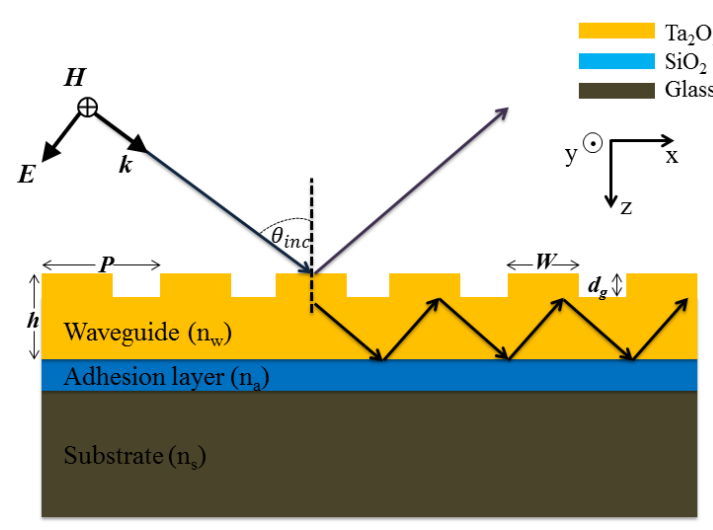

Fig. 1: Sketch of the proposed GRM filter. The grating layer is a rectangular profile with $d_{g}=$ thickness of grating, $W=$ grating width, $P=$ grating period. $n_{s}, n_{a}, n_{w}$ are refractive indices of the glass substrate, the $\mathrm{SiO}_{2}$ adhesion layer, and the homogeneous $\mathrm{Ta}_{2} \mathrm{O}_{5}$ waveguiding layer, respectively.

ing the phase-matching condition of the periodic structure [2]

$$
n_{e f f}=n_{c} \sin \theta_{i}-m \frac{\lambda}{P}
$$

where $n_{e f f}$ is the effective index of the equivalent homogeneous waveguide, $n_{c}$ is refractive index of air, $P$ is the grating period, $\lambda$ is the free space wavelength, $\theta_{i}$ is the incident angle, and the integer $\mathrm{m}$ represents the $\mathrm{m}^{\text {th }}$ diffracted order. Moreover, the condition for the guided wave to exist in the grating structure can be represented [2] as

$$
\max \left[n_{c}, n_{s}\right] \leq n_{e f f}<n_{w}
$$

The equation describes the regions of resonance for guided-mode resonance. At resonance wavelengths, part of the applied wave is coupled into a guided mode which gradually leaks out from the waveguide. The leaky-wave combines with the applied wave to generating a filtering response in the spectrum. That's why transmission dips appear at resonance wavelengths.

\section{SPECTROSCOPIC PROPERTY OF GMR FILTER}

The main goal of the proposed filter is to block a lightwave at a single wavelength while passing it at the others in the visible band of the electromagnetic spectrum. In this case, the transmission spectroscopy has an ultra-sharp transmission dip at the resonance and broadband transmission at the wavelengths away from the resonance. The resonant wavelength is tunable upon polarization states and angles of incidence. During the investigation, we observed that the periodicity (or pitch) of the structure plays an important role in positioning the transmission resonance at the normal incidence.

For simplification purposes, in Fig. 2, we only show the transmission features for various periodicities of the structure. The resonant transmission wavelength is linearly shifted with respect to the periodicity of the structure for the same structural parameters. For the case of $\mathrm{P}$ $=0.441 \mu \mathrm{m}$, the spectra shifted linearly toward short wavelength and a second dip simultaneously appears near the wavelength of $0.67 \mu \mathrm{m}$ in TE mode. Fig. 3 shows the transmission spectrum over the $0.5-0.8 \mu \mathrm{m}$ wavelength range at normal incidence for both TM and TE polarization states of the optimized filter structure which having the grating period of $0.49 \mu \mathrm{m}$, grating width of $0.16 \mu \mathrm{m}$, the grating thickness of 0.08 $\mu \mathrm{m}$ and the $\mathrm{Ta}_{2} \mathrm{O}_{5}$ waveguide layer of $0.1 \mu \mathrm{m}$.

For TM-polarized incidence, a single dip in the transmission spectrum is observed at $0.641 \mu \mathrm{m}$ as a stopband which corresponds to a low transmission less than 2\%, shown in Fig. 3(a). For TE-polarized incidence, the spectral response of the filter splits into two spectral dips comprising of a primary dip and a secondary dip located at the resonant wavelengths of $0.623 \mu \mathrm{m}$ and 0.737 $\mu \mathrm{m}$, respectively, shown in Fig. 3(b). The primary dip produces a transmission of less than $1 \%$.

Fig. 4 provides the transmission as a function of angles of incidence. In detail, Figs. 4(a) and 4(b) respectively show the calculated transmission spectra for various incident angles in TM 


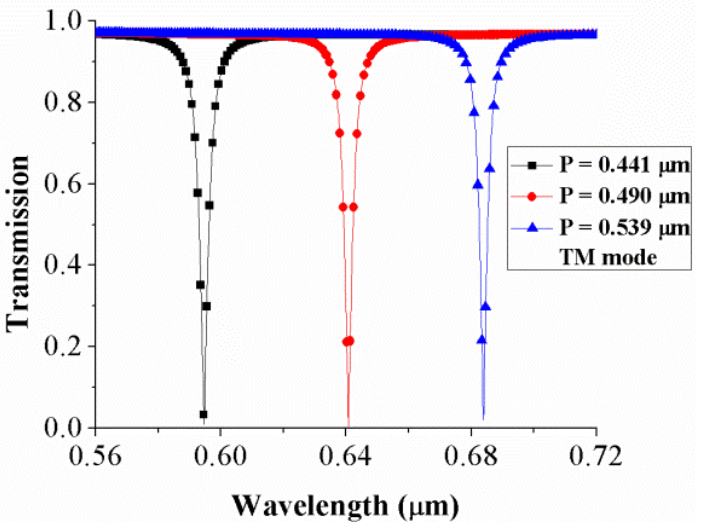

(a)

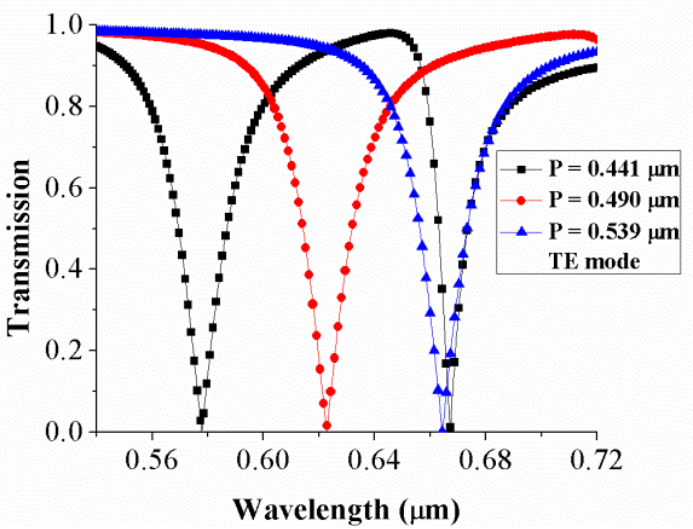

(b)

Fig. 2: The spectral transmission response of the GMR filter for various periodicities for TM mode (a) and TE mode (b) at normal incidence. The filter profile is described in Fig. 1 with parameters: $h$ $=0.1 \mu \mathrm{m}, d_{g}=0.08 \mu \mathrm{m}$, and $w=0.16 \mu \mathrm{m}$.

and TE polarizations. As the angles of incidence increase from 0 to 15 degrees, the transmission dip splits at $\lambda=641 \mathrm{~nm}$ which is clearly observed in Fig. 4(a) for TM-polarized light. Similarly, the transmission feature splitting at $\lambda=$ $737 \mathrm{~nm}$ and $\lambda=623 \mathrm{~nm}$ for TE-polarized light is also clearly observed in Fig. 4(b).

To highlight transmission features through our filter structure, we plot spatial distributions of the electric field at these resonant wavelengths for both polarization states. The amplitude magnitudes of the electric field $|\mathrm{Ey}|$ and of the magnetic field $|\mathrm{Hy}|$ for the both TE and TM mode in $\hat{y}$ direction are shown in Fig. 5. Fig. 5 (a) shows the total magnetic field distribution

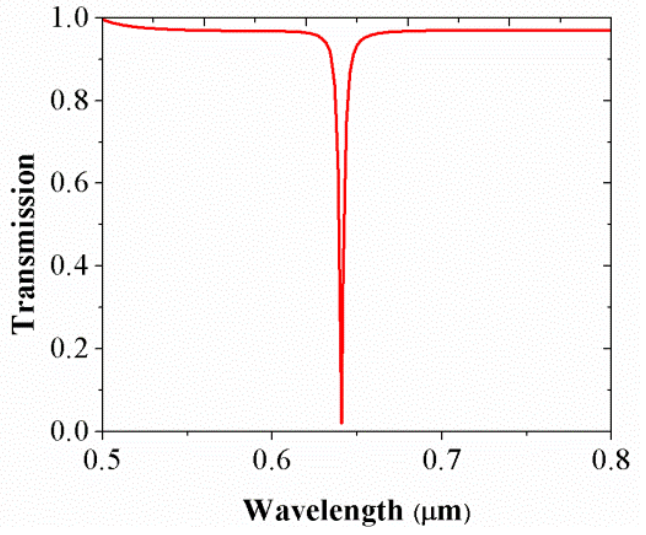

(a)

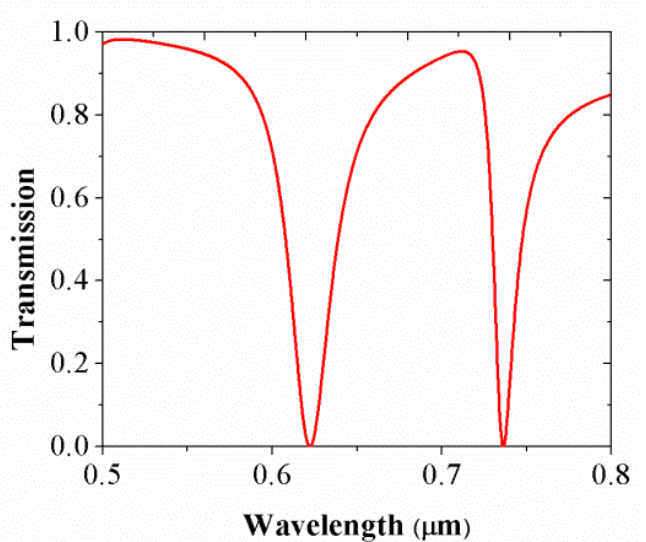

(b)

Fig. 3: Transmission spectra of the GMR filter. The resonance is due to the excitation of a TMpolarized guided mode (a) and a TE-polarized guided mode (b)

for TM normally incident light at $\lambda_{\text {res }}=641$ $\mathrm{nm}$. It is obvious that the field distribution, in this case, is located in the grating and disperses in the substrate. Similarly, Fig. 5(b) shows the total electric field distribution for TE normally incident light at $\lambda_{\text {res }}=623 \mathrm{~nm}$. The field distribution mainly concentrates on the grating interface and the substrate. The electric field enhancement inside the structure is due to the surface energy interference to the substrate from every grating period. Moreover, the coupled waves also take part in the surface energy interference and it has a greater value than the maximum electric field [22]. 


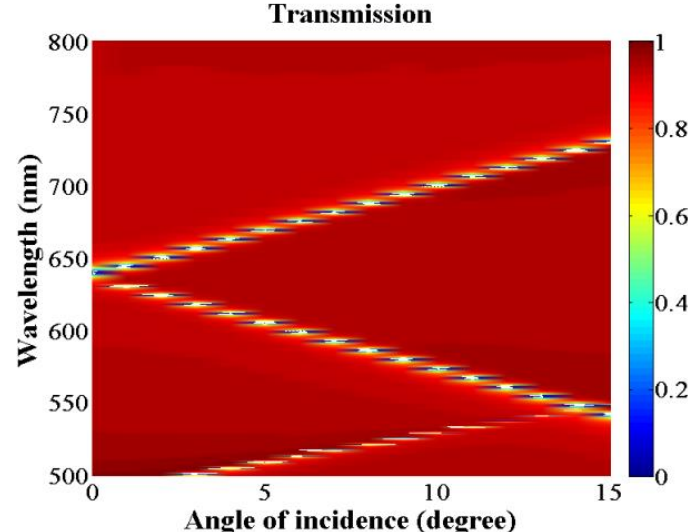

(a)

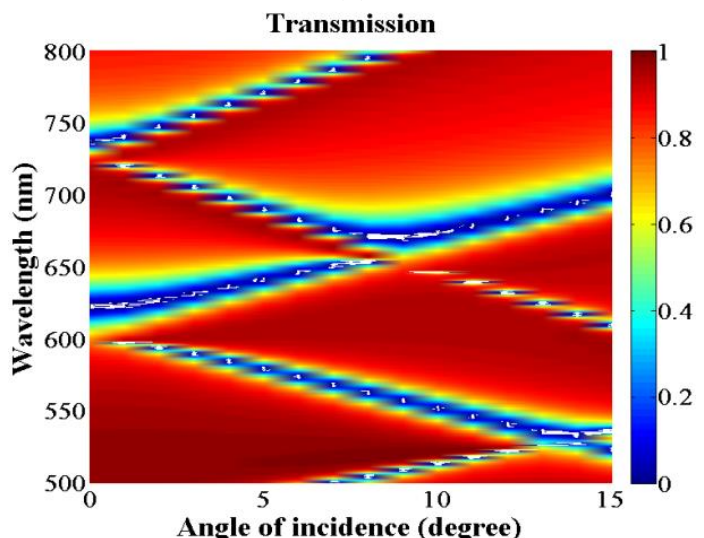

(b)

Fig. 4: Simulated transmission as a function of incident angle and wavelength for (a) TM polarization, and (b) TE polarization. The filter is sensitive to angles of incidence with a strong splitting of the transmission features.

\section{SURROUNDING REFRACTIVE INDEX EFFECT}

Owing to the GMR filter's narrow bandwidth and high sensitivity to the incidence environment, it is potential for sensing applications. In this section, we investigate a bulk refractive index sensing application of the proposed GMR filter in the visible band. Many works related to optical sensing applications have been proposed in previous publications [23-29]. Fig. 6 shows a resonant shift with respect to a change in the cover refractive index from $n=1$ to $n=1.3$ in the wavelength range of $0.6-0.7 \mu \mathrm{m}$ the both

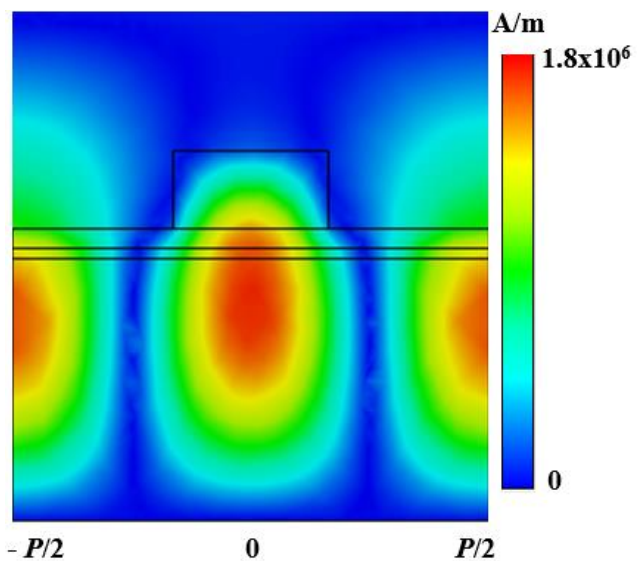

(a)

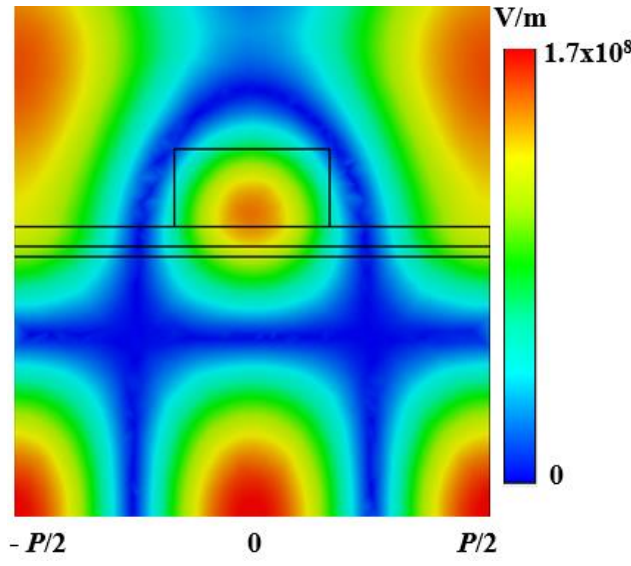

(b)

Fig. 5: Field distribution profiles for TM and TE associated with the transmission features at the resonant wavelengths. (a) $|\mathrm{Hy}|$ at $\lambda=0.641 \mu \mathrm{m}$ with incident TM-polarized in $\hat{y}$ direction. (b) |Ey $\mid$ at $\lambda=0.623 \mu \mathrm{m}$ with incident TE-polarized in $\hat{y}$ direction.

TM and TE polarizations. Beside the sensitivity $S$, another most important factor for sensing applications is the figure of merit (FOM) which is defined as a ratio between sensitivity and fullwidth at half-maximum (FWHM) centered at the resonant wavelength.

This factor is applied to further evaluate the sensing performance as the following relation, $\mathrm{FOM}=S / \mathrm{FWHM}[30]$, where $S=\delta \lambda / \delta n$ is the refractive index sensitivity (i.e., spectral shift per refractive index), $\delta \lambda$ and $\delta n$ are the variabil- 


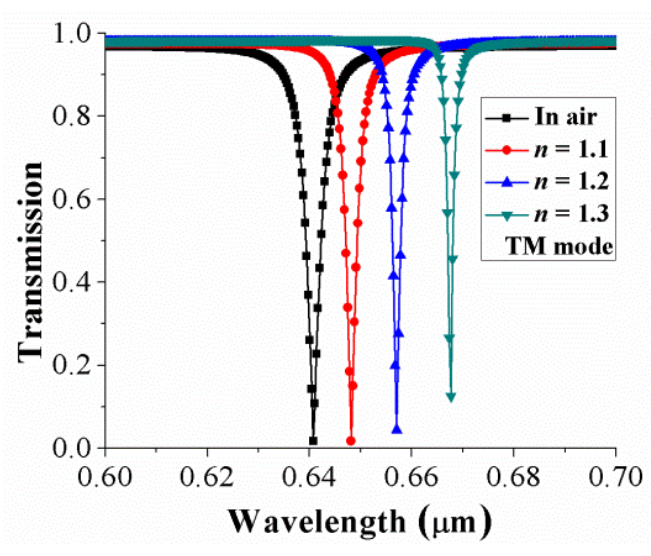

(a)

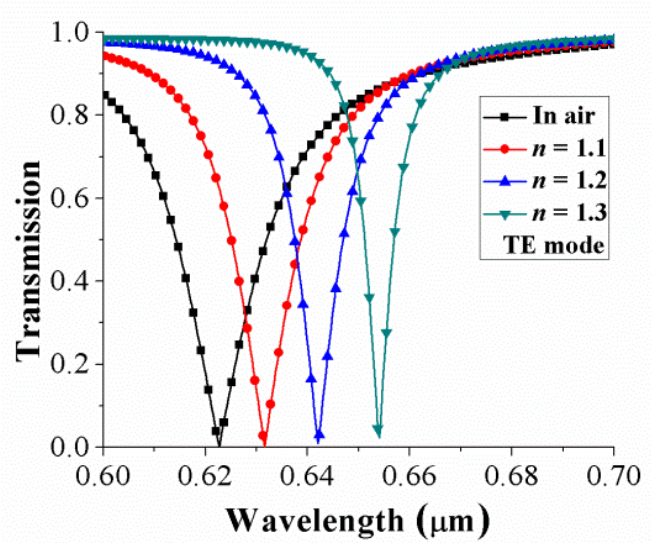

(b)

Fig. 6: Transmission dip shift with the refractive index of the surrounding environment varied from 1 to 1.3 for TM mode (a) and TE mode (b).

ity of wavelength and refractive index, respectively. The spectral properties of TM- and TEpolarized incident light with respect to the surrounding medium refractive index are presented in Table 1 and Table 2, respectively.

Generally, as the refractive index of the surrounding environment increases, the resonant wavelength at the transmission dip increases along with a corresponding increase of the quality factor (Q-factor $\left.=\lambda_{\text {res }} / \mathrm{FWHM}\right)$. In detail, when the refractive index of the surrounding environment increases, the resonant wavelength gradually shifts to near-infrared and the Q-factor of the transmission spectrum increases. For refractive indices higher than 1.3, almost
Tab. 1: Spectral properties of biosensing applications for TM polarization.

\begin{tabular}{|l|l|l|l|l|l|}
\hline \multicolumn{2}{|c|}{ Refractive index, $n$} & 1.0 & 1.1 & 1.2 & 1.3 \\
\hline $\begin{array}{l}\text { At } \\
\operatorname{dip}\end{array}$ & $\begin{array}{l}\text { Resonant } \\
\text { wavelength } \\
\text { (nm) }\end{array}$ & 641 & 648 & 657 & 668 \\
\cline { 2 - 6 } & $Q$-factor & 13.24 & 13.5 & 13.98 & 15.53 \\
\cline { 2 - 6 } & $\begin{array}{l}\text { Sensitivity } \\
\text { S (nm/RIU) }\end{array}$ & \multicolumn{4}{|c|}{ S $_{\text {average }}=90$} \\
\cline { 2 - 5 } & $\begin{array}{l}\text { Figure-of- } \\
\text { merit (FOM) }\end{array}$ & \multicolumn{4}{|c|}{ FOM $_{\text {average }}=1.93$} \\
\hline
\end{tabular}

Tab. 2: Spectral properties of biosensing applications for TE polarization.

\begin{tabular}{|c|c|c|c|c|c|}
\hline \multicolumn{2}{|c|}{ Refractive index, $n$} & 1.0 & 1.1 & 1.2 & 1.3 \\
\hline \multirow[t]{4}{*}{$\begin{array}{l}\text { At } \\
\text { dip }\end{array}$} & $\begin{array}{l}\text { Resonant } \\
\text { wavelength } \\
(\mathrm{nm})\end{array}$ & 623 & 632 & 642 & 654 \\
\hline & $Q$-factor & 12.71 & 12.98 & 13.24 & 13.57 \\
\hline & $\begin{array}{l}\text { Sensitivity } \\
S(\mathrm{~nm} / \mathrm{RIU})\end{array}$ & \multicolumn{4}{|c|}{$\mathrm{S}_{\text {average }}=103.33$} \\
\hline & $\begin{array}{l}\text { Figure-of- } \\
\text { merit (FOM) }\end{array}$ & \multicolumn{4}{|c|}{ FOM $_{\text {average }}=2.13$} \\
\hline
\end{tabular}

all mediums are solids and liquids, therefore Q-factor will increase with falling of FWHM. The calculated sensitivity and FOM are about $90 \mathrm{~nm} / \mathrm{RIU}$ and 1.93 for TM polarization while about $103.33 \mathrm{~nm} / \mathrm{RIU}$ and 2.13 for TE polarization. Obviously, for the same structure, TE polarization results in higher sensitivity and FOM. In comparison between the spectral properties of biosensing applications for the both TM and TE polarizations at normal incidence, the state of TE polarized light has a better performance in terms of sensitivity and FOM in the same wavelength range. Each of polarization states will create its own resonant transmission dips occurring at different wavelengths. Therefore, the proposed GMR filter primarily works as a wavelength selective polarizer. Depending on the state of polarization of incident light and the refractive index of the cover medium, the ideal sensor should be based on TE mode with greater $\mathrm{S}_{\text {average }}$ and $\mathrm{FOM}_{\text {average }}$ to fabricate. 


\section{CONCLUSIONS}

We have presented an ultra-narrowband filter operating in the visible band of the electromagnetism spectrum. The filter almost blocks the lightwave transmission at the resonant wavelength while passing it over the remaining wavelengths out of the resonance for both TM and TE polarization states. The transmission features can be amended by adjusting the incident angles, the polarization of incident light, and the refractive index of the surrounding environment. Such selective characteristics of the proposed GMR filter meet demands for practical applications such as tunable optical filters, various imaging or detection, and refractive index sensing.

\section{References}

[1] Magnusson, R., \& Wang, S.S. (1992). New principle for optical filters. Appl. Phys. Lett., 61 (9), 1022.

[2] Wang, S.S., \& Magnusson, R. (1993). Theory and applications of guided-mode resonance filters. Appl. Opt., 32(14), 26062613.

[3] Popov, E., Mashev, L., \& Maystre, D. (1986). Theoretical study of the anomalies of coated dielectric gratings. J. Mod. Opt., $33(5), 607-619$.

[4] Lacour, D., Granet, G., Plumey, J.P., \& Mure-Ravaud, A. (2003). Polarization independence of a one-dimensional grating in conical mounting. J. Opt. Soc. Am. A, 20(8), 1546-1552.

[5] Niederer, G., Nakagawa, W., Herzig, H.P., \& Thiele, H. (2005). Design and characterization of a tunable polarizationindependent resonant grating filter. Opt. Expr., 13(6), 2196-2200.

[6] Peters, D.W., Boye, R.R., Wendt, J.R., Kellogg, R.A., Kemme, S.A., Carter, T.R., \& Samora, S. (2010). Demonstration of polarization-independent resonant subwavelength grating filter arrays. Opt. Lett., 35 (6), 3201-3203.

[7] Wang, S.S., \& Magnusson, R. (1994). Design of waveguide-grating filters with symmetrical line shapes and low sidebands. Opt. Lett., 19(12), 919-921.

[8] Sang, T., Cai, S., \& Wang, Z. (2011). Guided-mode resonance filter with an antireflective surface consisting of a buffer layer with refractive index equal to that of the grating. J. Mod. Opt, 58(14), 12601268.

[9] Liu, Z.S., Tibuleac, S., Shin, D., Young, P.P., \& Magnusson, R. (1998). Highefficiency guided-mode resonance filter. Opt. Lett., $23(19)$, 1556-1558.

[10] Quan, H. \& Guo, Z. (2005). Simulation of whispering-gallery-mode resonance shifts for optical miniature biosensors. J. Quant. Spectrosc. Radiat. Transf., 93(1), 231-243.

[11] Lee, K.J., \& Magnusson, R. (2011). SingleLayer Resonant High Reflector in TE Polarization: Theory and Experiment. IEEE. Photon. J, 3(1), 123-129.

[12] Zhong, Y., Goldenfeld, Z., Li, K., Streyer, W., Yu, L., Nordin, L., Murphy, N., \& Wasserman, D. (2017). Mid-wave infrared narrow bandwidth guided mode resonance notch filter. Opt. Lett., 42(2), 223-226.

[13] Zeng, G., Zou, X., Xu, L., \& Wang, J. (2017). Single layer narrow bandwidth angle-insensitive guided-mode resonance bandstop filters. Optik, 130, 19-23.

[14] Chang-Hasnian, C.J., \& Yang, W. (2012). High-contrast gratings for integrated optoelectronics. Adv. Opt. Photon., 4(3), 379440 .

[15] Akahane, Y., Asano, T., Song, B.S., \& Noda, S. (2003). High-Q photonic nanocavity in a two-dimensional photonic crystal. Nature, 425, 944-947.

[16] Golubenko, G.A., Svakhin, A.S., Sychugov, V.A., \& Tishchenko, A.V. (1985). Total reflection of light from a corrugated surface 
of a dielectric waveguide. Sov. J. Quantum. Electron., 15, 886-887.

[17] Ngo, Q.M., Le, K.Q., \& Vu, D.L. (2012). Optical bistability based on guided-mode resonances in photonic crystal slabs. J. Opt. Soc. Am. B., 29(6), 1291-1295.

[18] Le, K.Q., Ngo, Q.M., \& Nguyen, T.K. (2017). Nanostructured metal-insulatormetal metamaterials for refractive index biosensing applications: Design, fabrication, and characterization. IEEE. J. Sel. Topics. Quantum. Electron., 23(2), 6900506.

[19] Le, K.Q., Bai, J., Ngo, Q.M., \& Chen, P.Y. (2016). Fabrication and Numerical Characterization of Infrared Metamaterial Absorbers for Refractometric Biosensors. $J$. Electron. Mater., 46(1), 668-676.

[20] Vo-Dinh, T., Griffin, G., Stokes, D.L., Stratis-Cullum, D.N., Askari, M., \& Wintenberg, A. (2004). Optical sensors: Industrial environmental and diagnostic applications. Berlin: Springer.

[21] CST GmbH, CST Microwave Studio. (2016). Retrieved from http://www.cst.com

[22] Sun, T., Ma, J., Wang, J., Jin, Y., He, H., Shao, J., \& Fan, Z. (2008). Electric field distribution in resonant reflection filters under normal incidence. J. Opt. A: Pure. Appl. Opt., 10, 125003.

[23] Bottazzi, B., Fornasari, L., Frangolho, A., Giudicatti, S., Mantovani, A., Marabelli, F., Marchesini, G., Pellacani, P., Therisod, R., \& Valsesia, A. (2014). Multiplexed label-free optical biosensor for medical diagnostics. J. Biomed. Opt., 19(1), 17006.

[24] Lu, X., Zhang, L., \& Zhang, T. (2015). Nanoslit-microcavity-based narrow band absorber for sensing applications. Opt. Expr., 23(16), 20715-20720.

[25] Shen, Y., Zhou, J., Liu, T., Tao, Y., Jiang, R., Liu, M., Xiao, G., Zhu, J., Zhou, Z.K.,
Wang, X., Jin, C., \& Wang, J. (2013). Plasmonic gold mushroom arrays with refractive index sensing figures of merit approaching the theoretical limit. Nat. Commun., 4, 2381.

[26] El-Gohary, S.H, Choi, J.M, Kim, N.H., \& Byun, K.M. (2014). Plasmonic metaldielectric-metal stack structure with subwavelength metallic gratings for improving sensor sensitivity and signal quality. Appl. Opt., 53(10), 2152-2157.

[27] Wang, Y., Kar, A., Paterson, A., Kourentzi, K., Le, H., Ruchhoeft, P., Willson, R., \& Bao, J. (2014). Transmissive Nanohole Arrays for Massively-Parallel Optical Biosensing. ACS. Photonics, 1(3), 241-245.

[28] Mayerhöfer, T.G., Knipper, R., Hübner, U., Cialla-May, D., Weber, K., Meyer, H.G., \& Popp, J. (2015). UltraSensing by Combining Extraordinary Optical Transmission with Perfect Absorption. ACS. Photonics., 2(11), 1567-1575.

[29] Lu, X., Wan, R., \& Zhang, T. (2015). Metal-dielectric-metal based narrow band absorber for sensing applications. Opt. Expr., 23(23), 29842-29847.

[30] Le, K.Q., Alù, A., \& Bai, J. (2015). Multiple Fano interferences in a plasmonic metamolecule consisting of asymmetric metallic nanodimers. J. Appl. Phys., 117(2), 023118 .

\section{About Authors}

Phuc Toan DANG received Bachelor's degree in Physical pedagogy from An Giang University, Vietnam, in 2012, and Master's degree in Theoretical and mathematical physics from Can Tho University, Vietnam, in 2016. From 2016 to 2019, he joined the Institute for Computational Science, Ton Duc Thang University, as an assistant researcher. Since 2019 to present, he has been a PhD. sdtudent in Display Engineering Lab, Faculty of Electronics and Information Engineering, Chonbuk National University. His current research interests include solar cells, 
liquid crystal, dielectric spectroscopy, optics and photonics.

Khai Q. LE has more than 10 years of experience in nanophotonics, who is in charge of design, fabrication and characterization of subwavelength optical components. He received his Ph.D. in Photonics Engineering at Ghent University, Belgium in 2011. He conducted his postdoctoral research in several leading academic institutions including University of Texas at Austin, USA, University of Toronto, Canada, University of Minnesota Duluth, USA and Institute for Molecular Science, Japan. He has recently switched to the industry working as a nanofabrication engineer at the aBeam Technologies, Inc. His main tasks are related to the photonic design, nanofabrication and optical measurements. He has published 65 scientific papers (cited over 1080 times) in peerreviewed high-impact factor journals. He is currently also affiliated with Lawrence Berkeley National Lab (LBNL), USA and Ton Duc Thang University, Vietnam.

Quang Minh NGO received his $\mathrm{PhD}$ in electrical engineering at Ajou University, the Republic of Korea in 2011. From March 2012 to January 2019, he had worked as the leader of micro- and nanophotonics research group at Institute of Materials Science (IMS), Vietnam Academy of Science and Technology (VAST), Hanoi, Vietnam. Since February 2019, he has worked as the director of Administration, University of Science and Technology of Hanoi (USTH), VAST, Hanoi, Vietnam. He is an author/coauthor of 50 ISI papers. His main research focuses on design, simulation, fabrication and characterization of micro- and nanophotonics in the visible and near-infrared spectral regions for optical devices.

P. T. H. NGUYEN is an assistant professor in the Department of Electrical and Computer Engineering at New Jersey Institute of Technology (NJIT), U.S. He received B.S. degree in Physics from Vietnam National University in Ho Chi Minh City, Vietnam (2005), the M.S. degree in Electronics Engineering from Ajou University, South Korea (2009), and the PhD. degree in Electrical Engineering from McGill
University, Canada (2012). He is the founder and director of the molecular beam epitaxy facility at NJIT. His current research interests include molecular beam epitaxial growth, fabrication, and characterization of III-V nanowire heterostructures for high-performance nanooptoelectronic devices including LEDs, lasers, photodetectors, solar fuels, and solar cells. Dr. Nguyen is the author/coauthor of more than 55 journal articles and 90 conference presentations. He was a recipient of the SPIE scholarship in optics (2012), the best student paper award (2nd place) at the IEEE Photonics conference (2011), and the outstanding student paper award at the 28th North American Molecular Beam Epitaxy conference (2011).

Truong Khang NGUYEN received the B.S. degree in Computational Physics from the University of Science, Vietnam National University, Ho Chi Minh City in 2006, and the M.S. and Ph.D. degrees in Electrical and Computer Engineering from Ajou University in Suwon, Korea in 2013. From Oct. 2013 to Dec. 2014, he worked at Division of Energy Systems Research, Ajou Univerisity, Korea as a postdoctoral fellow. He is currently Head of Division of Computational Physics at Institute for Computational Science, Ton Duc Thang University in Ho Chi Minh City, Vietnam, and also Managing Editor of Journal of Advanced Engineering and Computation. He has authored and co-authored 60 peer-reviewed ISI journal articles and 40 conference papers. He has written one book chapter in the area of terahertz antenna and filed one patent on terahertz stripline antenna. His current research interests include Microwave Antenna for Wireless Communication; Terahertz Antenna for Compact and Efficient Source; Nano Structures and Nano Antenna for Optical Applications; and Computational Micro/Nano Fluidics. 